\title{
Laparoscopic Splenectomy in Non-Cirrhotic Portal Hypertension- A Retrospective Analysis of a Prospectively Performed Series
}

\author{
Rajkumar J S, Jayakrishna Reddy Aluru*, Anirudh Rajkumar, Shreya Rajkumar, Dharmendra K R and Nabeel \\ Nazeer
}

Junior Consultant, Lifeline Rigid Hospital, India

Submission: December 26, 2020; Published: January 07, 2020

*Corresponding author: Jayakrishna Reddy Aluru, Junior Consultant, Lifeline Rigid Hospital, 47/3 New Avadi Road, Kilpauk, Chennai-600010, India

Abstract

In our institute, laparoscopic splenectomy was performed in 27 patients over a period of 7 years for two major indications: hypersplenism and refractory variceal bleeding. 19 patients had Extra Hepatic Portal Venous Obstruction (EHPVO) and 8 patients had Non-Cirrhotic Portal Fibrosis ( NCPF). All the patients had hypersplenism, with thrombocytopenia( $<50,000 / \mathrm{cu} . \mathrm{mm}$ ),leukopenia $(<4000 / \mathrm{Cu}$ mm)as well in 9 (33\%)of the patients, and anemia ( $\mathrm{Hb}<10 \mathrm{gm})$ in $8(30 \%)$. Variceal bleeding requiring Endoscopic Variceal Ligation (EVL) were found in 23 patients, 17 in the EHPVO group and 6 in the NCPF group . 4 patients were females and 23 were male. The age range was from 12 to 37 years, the mean being 24 years. The laparoscopic procedure was successful in 25 , but 2 patients needed conversion to left subcostal laparotomy because of extensive and giant collaterals around the hilum of the spleen; these conversions happened in the first three years of our laparoscopic splenectomy experience, with no conversions in the subsequent 4 years. Even in the presence of a relative contraindication like portal hypertension, laparoscopic splenectomy is still a viable proposition in the vast majority of cases. Technical considerations, like deployment of powerful energy sources, vascular staplers and preliminary splenic artery ligation are discussed in this article.

Keywords: Splenectomy; Endoscopy variceal ligation; Extra hepatic portal venous obstruction; Hypersplenism; Non-cirrhotic portal fibrosis; Portal hypertension

\section{Introduction}

Apart from cirrhosis, prehepatic portal hypertension is quite common in the authors' part of the world, the two major varieties being Extra Hepatic Portal Venous Obstruction(EHPVO) and NonCirrhotic Portal Fibrosis(NCPF). Although managed usually with endoscopic variceal ligation on an out-patient basis, some of these patients required surgical intervention because of hypersplenism. Over the past 7 years, all such patients who underwent laparoscopic splenectomy in our institution were studied. This paper constitutes a retrospective analysis of a prospectively performed series.

\section{Materials and Methods}

\section{Inclusion criteria}

a. All Extra Hepatic portal hypertension patients presenting with hypersplenism were included.

b. Exclusion criteria: c. Massive splenomegaly (defined as beyond the umbilicus)

d. Liver cirrhosis

e. Associated chronic pancreatitis

f. Previous surgeries in the upper abdomen

g. Emergency devascularization

\section{Methods}

None of the patients had preoperative coeliac angiography or embolization. Shunting was not done in any patients. All patients had polyvalent pneumococcal vaccine and $\mathrm{H}$. influenza vaccine. Pre-operative antibiotics (Cefuroxime)were given. Postoperatively Low Molecular Weight Heparin (LMWH) was given for five days.

Data was analyzed for the following:

a. Ease of laparoscopic completion 
b. Time taken

c. Learning curve definition

d. Number of days in hospital

e. Conversion and reasons

f. Post-splenectomy complications in the 30-day period

\section{Technique}

A right lateral position was used for all. A four-port approach was used , all surrounding the left costal margin in a gentle concave curve. A $10 \mathrm{~mm}$ Camera port was inserted, 2 to 3 inches from the left costal margin along a line drawn from the umbilicus to the left costal margin, at the inter-section of the left mid clavicular line. Left-hand and right-hand working ports were put in, at four fingerbreadths distance from the camera port, on either side. A fourth port for retraction/posterior dissection was put in, further down at the inter-section of the left costal margin with the posterior axillary line (Figure 1). Any additional retraction was performed using a Verses needle covered by the plastic hub of the intravenous line needle. Diagnostic laparoscopy was done, including liver status and biopsy, and checking for splenunculi.

Figure 1: Ports placement.

Throughout, the posterior attachments of the spleen to the kidney and diaphragm(the posterior layer of the splenorenal ligament and the Lienophrenic ligament) were left in place during the dissection, to be released as the final step. This is known as the "hanging clock" technique, permitting the spleen to stay attached posteriorly. The first step was to identify the number of splenic notches along its border. Multiple notches indicate a "distributed" pattern of the splenic artery, and a single notch indicates a "magisterial" type of splenic artery. Based on the notches, the gastrocolic ligament was opened, either proximally to take a single artery (24 patients) or more distally to take multiple small branches (3 patients).

The splenic artery was ligated at the upper border of the pancreas, after gently dissecting its peritoneal covering with a right-angled instrument and silk suture (Figure 2). The gastrosplenic ligament and the short gastric vessels were then taken down with the ultrasonic shears, staying close to the spleen (Figure 3). The Ligasure was also found to be very useful, especially for larger vessels. Inferior attachments to the colon were then released. Multiple spontaneous lienorenal collaterals were left alone during this part of the dissection. In two patients
, severe bleeding from these collaterals engendered conversion to open surgery.

Then the anterior layer of the splenorenal ligaments was taken down to expose the splenic vessels at the hilum of the spleen and tail of the pancreas. The latter was dissected off from the hilar vessels, to avoid pancreatic injury. The splenic artery was ligated first, which resulted in complete decongestion and pultaceous appearance of the spleen, followed by splenic vein ligation. The hilum was taken down with multiple ties in 25 patients, with a vascular stapler being used in two patients (Figure 4). After the medial portion of the spleen was freed completely, the posterior dissection was completed using the fourth (lateral most) port. The spleen was bagged and removed through a Pfannenstiel incision.

\section{Results}

Only two cases required conversion to open surgery. The others were successfully completed laparoscopically. The operating time for completion was analyzed using ANOVA (Table 1). The average blood loss was $150 \mathrm{ml}$. Perioperative blood transfusion was needed only in the 2 patients who required conversion to open surgery. 


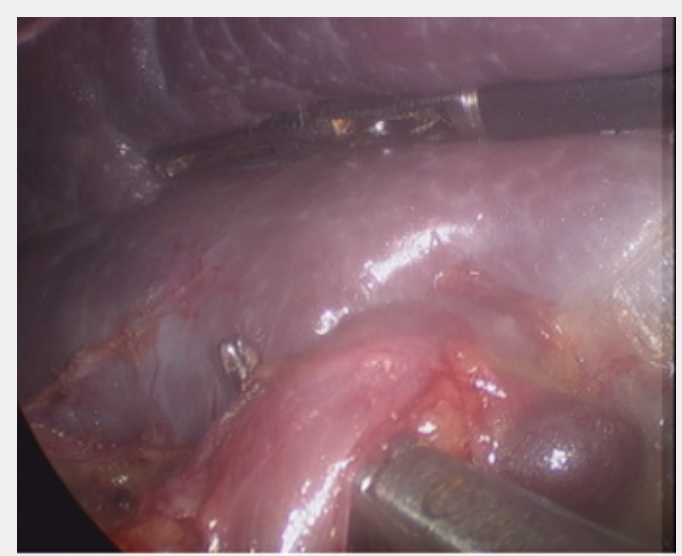

Figure 2: Splenic artery dissection.

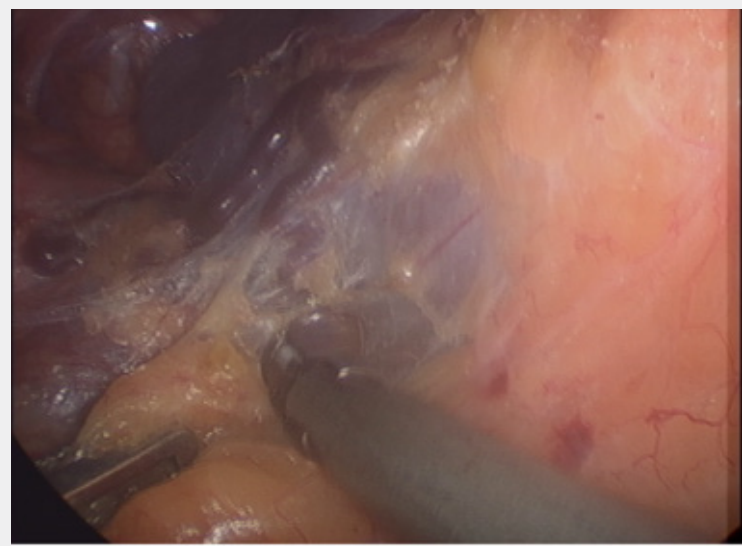

Figure 3: Gastrosplenic ligament release.

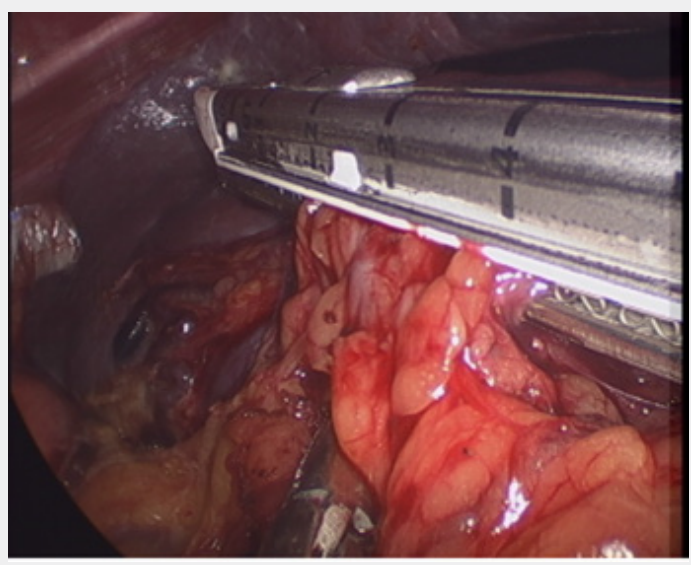

Figure 4: Vascular Stapler.

Table 1: One-way Analysis of Variance (ANOVA).

\begin{tabular}{|c|c|c|c|c|c|}
\hline Group & No of Patients & Mean Time & Standard Deviation Time & Standard Error of Mean Time & Median Time \\
\hline $1^{\text {st }}$ & 9 & 169.67 & 37.944 & 12.648 & 157 \\
\hline $2^{\text {nd }}$ & 9 & 111.33 & 31.06 & 10.353 & 110 \\
\hline $3^{\text {rd }}$ & 9 & 73.44 & 14.518 & 4.839 & 70 \\
\hline
\end{tabular}


There was no immediate or 30-day mortality, and no morbidity, either to surgery or to blood transfusions in the two converted patients. In the first nine patients there were two conversions , and none thereafter. The average post-operative length of stay was 4.5 days, with conversions patients staying for 7 and 9 days.

Follow-up:

$$
\begin{aligned}
& \text { a) } 1 \text { month(27 patients) } \\
& \text { b) } 3 \text { months (27 patients) } \\
& \text { c) } 12 \text { months (22 patients) } \\
& \text { d) } 24 \text { months (12 patients) }
\end{aligned}
$$

There were no patients with post-splenectomy infections or deep vein thrombosis. All blood parameters improved significantly by 3 months follow-up. Analysis of time taken clearly indicates a learning curve, probably a combination of experience, more powerful energy sources, and vascular staplers. The liver biopsies showed normal liver architecture in 19 (EHPVO) and mild periportal fibrosis (NCPF)in 8. Long-term follow-up was possible in $12(44 \%)$ patients only. The rest were lost to follow-up.

\section{Discussion}

Since the first laparoscopic splenectomy was reported in 1991, LS has become the gold standard for the removal of normal to moderately enlarged spleens in benign conditions [1]. Laparoscopic splenectomy is a straightforward procedure for diseases like Idiopathic Thrombocytopenic purpura (ITP) and spherocytosis. On the other hand, even open splenectomy may be a nightmare for surgeons, especially because of collaterals causing possibly fatal bleeding [2].

It was found that primary ligation of the splenic artery (which supplies $50 \%$ of total circulation) made the procedure much simpler. Utilizing newer energy sources like the Harmonic scalpel or the Ligasure also enabled a bloodless field around the spleen [3]. In two difficult cases, using the vascular stapler permitted completion of hilar vascular transection [1]. Adopting the hanging clock manoeuvre permitted accurate pericapsular dissection of the spleen. Staying close to the capsule of spleen helped avoid the splenorenal collaterals, and unnecessary intra operative hemorrhage. Combining all the techniques mentioned above made facile an otherwise technically demanding operation.

According to L R Elkief, et al., patients with Idiopathic NonCirrhotic Portal Hypertension (INCPH), performed retrospective analyses of patients with INCPH undergoing abdominal surgery [4]. X Chen, et al., reviewed revealed laparoscopic splenectomy and laparoscopic splenectomy with devascularization to be safe and effective in the setting of liver cirrhosis and portal hypertension. From the comparison articles, laparoscopic procedures appear to be superior to open procedures regarding blood loss, hospital stay, complication rate and liver function impairment [5]. R Rajalingam et al. concluded that Hypersplenism is effectively relieved by both shunt and non-shunt operations. A proximal splenorenal shunt not only relieves hypersplenism but also effectively addresses the potential complications of underlying portal hypertension and can be safely performed with good long-term outcome [6].

Laparoscopic splenectomy is being done for cirrhotics, with hypersplenism, and viral cirrhotics on interferon. A single case report of laparoscopic splenectomy for left-sided portal hypertension, preceded by pre-operative embolization, reported by Patrono et al. [7]. A comprehensive study by ZHAN et al, in the World Journal of Gastroenterology on laparoscopic splenectomy for hypersplenism secondary to cirrhosis and portal hypertension, [1] also concluded that this was quite a safe operation for this group of patients.

\section{Conclusion}

This paper reviews a single surgical team, single institution series of laparoscopic splenectomy in non-cirrhotic portal hypertension. The procedure is feasible and safe in experienced hands, with the use of assistive devises ,has a definite learning curve, seems facilitated by prior splenic artery ligation, and required conversion in less than $10 \%$.

\section{References}

1. Zhan XL, Ji Y, Wang YD (2014) Laparoscopic splenectomy for hypersplenism secondary to liver cirrhosis and portal hypertension. World J Gastroenterol 20 (19): 5794-800.

2. Yang K, Yun SP, Kim JH, Kim DH, Kim HS, et al. (2017) Laparoscopic Splenectomy for a Patient with Splenomegaly and Hypersplenism due to Idiopathic Portal Hypertension. J Minim Invasive Surg 20(3): 113116.

3. Mingjun Wang, Xin Wang, Hua Zhang, Yunqiang Cai, Bing Peng (2015) Laparoscopic splenectomy for portal hypertension secondary to liver cirrhosis: Ligasure combined with ultrasound scalpel versus ultrasound scalpel - SAGES Abstract Archives.

4. RElkief L, Ferrusquia Acosta J, Payancé A, Moga L, Tellez L, et al. (2019) Abdominal Surgery in Patients With Idiopathic Noncirrhotic Portal Hypertension: A Multicenter Retrospective Study. Hepatology 70(3): 911-924.

5. Chen X D, He F Q, Yang L, Yu Y Y, Zhou Z G (2013) Laparoscopic splenectomy with or without devascularization of the stomach for liver cirrhosis and portal hypertension: a systematic review. ANZ J Surg 83(3): 122-128.

6. Rajalingam R, Javed A, Sharma D, Sakhuja P, Singh S, et al. (2012) Management of hypersplenism in non-cirrhotic portal hypertension Hepatobiliary Pancreat Dis Int 11(2): 165-171.

7. Patrono D, Benvenga R, Moro F, Rossato D, Romagnoli R, et al. (2014) Left-sided portal hypertension: Successful management by laparoscopic splenectomy following splenic artery embolization. Int J Surg Case Rep 5(10): 652-655. 
This work is licensed under Creative Commons Attribution 4.0 License DOI:10.19080/ARGH.2021.16.555937

\section{Your next submission with JuniperPublishers} will reach you the below assets

- Quality Editorial service

- Swift Peer Review

- Reprints availability

- E-prints Service

- Manuscript Podcast for convenient understanding

- Global attainment for your research

- Manuscript accessibility in different formats ( Pdf, E-pub, Full Text, audio)

- Unceasing customer service

Track the below URL for one-step submission https://juniperpublishers.com/online-submission.php 